\title{
Identifying risk factors for graft loss within 90 days of kidney transplantation in the modern era: A review of single center and UNOS databases
}

\author{
Joseph T. Brooks ${ }^{1 *}$, Graham Mitro ${ }^{1}$, Kevin Becker ${ }^{1}$, Natasha Ahuja ${ }^{1}$, Bilel Gdoura ${ }^{2}$, Naoru Koizumi ${ }^{2}$, Michael Rees ${ }^{1}$ and Jorge Ortiz $^{1}$ \\ ${ }^{1}$ University of Toledo College of Medicine and Life Sciences, Toledo, OH, USA \\ ${ }^{2}$ George Mason University, Arlington, VA, USA
}

\begin{abstract}
Aim: Our aim was to clarify the risk factors for graft loss within the first 90 days of kidney transplantation.

Methods: We performed an IRB-approved, retrospective review of the United Network for Organ Sharing database (2010-2015) and our own single center database (2004-2015). We analyzed risk factors for early graft loss (EGL). EGL was defined as graft loss due to patient death, graft thrombosis, acute rejection, or primary non-function within 90 days of transplantation.

Results: At our center, 30 of 676 recipients experienced EGL (4.4\%). The most common cause of EGL at our center was recipient death. Demographic variables associated with EGL included: expanded criteria donor $(\mathrm{p}<0.001)$, older donors $(\mathrm{p}=0.003)$, donors with higher $\mathrm{BMI}(\mathrm{p}=0.004)$, and higher KDPI ( $\mathrm{p}=0.001)$. One, 3-, and 5-year patient survival was lower in recipients with EGL (all $\mathrm{p}<0.001$ ). Multivariate analysis suggested expanded criteria donor and donor BMI were predictors of EGL $(\mathrm{p}<0.001)$.

The rate of EGL among patients in the UNOS database was 3.35\%, with patient death being the most common cause. Multivariate analysis of the UNOS database revealed only recipient age was a predictor of EGL due to patient death $(\mathrm{p}<0.001)$. There were no predictors of EGL due to thrombosis. Previous kidney transplant and recipient age were predictors of EGL due to acute rejection ( $\mathrm{p}=0.002$ and $\mathrm{p}<0.001)$.
\end{abstract}

Conclusions: Overall between our center and UNOS database, patient death was the most common cause of EGL. Single center and UNOS data suggest that EGL occurs more frequently in recipients of sub-optimal allografts. Additionally, older age and previous transplant is associated with increased risk for EGL.

\section{Introduction}

Kidney transplantation is the most effective treatment available for patients with end-stage renal disease (ESRD). However, in 2014, only 17,107 kidney transplants were performed in the United States leaving over 80,000 people waiting for a transplant [1]. Because of the need for more donors, there has been increasing utilization of suboptimal kidneys, which are associated with inferior outcomes [2,3]. Additionally, it has been suggested that the use of these allografts is linked to early graft loss (EGL) [4].

EGL is defined as graft loss occurring within 90 days of kidney transplantation. It is relatively rare, occurring after approximately $5 \%$ of transplantations [5]. Causes of EGL include: patient death, renal artery or vein thrombosis, acute rejection (AR), and primary non-function (PNF). Very short-term outcomes after kidney transplantation are understudied. Additionally, there have been few reports on risk factors for EGL, and those that have been published are limited to single center analyses $[4,5]$.

Thus, our aim was to clarify those risk factors. Additionally, we sought to determine if there was a difference in the risk factors associated with each specific cause of EGL. We utilized the United Network for Organ Sharing (UNOS) database and our own single center database for our analysis. We hypothesized that the rate of EGL nationwide would be similar to that reported in prior single center analyses.

\section{Materials and methods}

\section{UNOS database}

Data containing kidney transplant donor and recipient $(n=56,883)$ information from June 6, 2011 through June 30, 2015 was extracted from the UNOS database. Recipients of living donor organs and simultaneous liver, pancreas, intestine, lung or heart transplants were excluded. Details regarding the recipient and donor demographics were queried. Recipient characteristics obtained included age, gender, BMI, time on waitlist, dialysis status, clinical status (ICU vs hospitalized vs home) and mechanical ventilatory needs at time of transplantation. Donor characteristics obtained included age, gender, race, BMI, creatinine, bilirubin, donor risk index (DRI), expanded criteria donor (ECD), and donation after cardiac death (DCD). Additional perioperative details were queried, including human leukocyte antigen (HLA) mismatch level, warm ischemia time, and cold ischemia time.

Correspondence to: Joseph Brooks, 5227 Carlingfort Dr Toledo, OH 43623, USA, E-mail: Joseph.brooks@rockets.utoledo.edu

Key words: early graft loss, kidney transplantation

Received: July 10, 2017; Accepted: August 22, 2017; Published: August 25, 2017 


\section{Study population and variables}

Primary outcome of this study was rate of EGF due to any cause at 7-, 30-, and 90-days post-transplant. Causes of EGF were further analyzed and classified into primary graft dysfunction/nonfunction, graft thrombosis (renal artery and vein), acute and chronic rejection, or others. Secondary outcome includes patient death from any cause.

\section{Statistical analysis}

Descriptive statistics of clinical and demographic characteristics were summarized using one-way ANOVA and t-test for continuous variables and chi-square test for categorical variables. Missing variables were omitted from the statistical analysis. For continuous variables, compliance with the normality assumption was tested using ShapiloWilk diagnostic test, and Kruskal Wallis rank test was performed when the normality assumption was violated. Patients who did not experience any of the end points were censored on the last follow-up date or on June $30^{\text {th }}, 2015$. Multivariate Cox-regression analyses were conducted to investigate adjusted risk factors. Those included donor and recipient demographics, peri- and post-operative factors, factors linked to organ quality, and organ share type (local, regional, or national).

\section{Single center database}

We analyzed our database of 676 patients who received kidney transplants and were induced with Alemtuzumab (ALE) at the University of Toledo Medical Center in Toledo, Ohio between March 2004 and November 2015. Patient data was reviewed using TransChart electronic medical record software (TransChart LLC, Dublin, Ohio). Donor information included: sex, age, ethnicity, presence of hypertension, presence of diabetes mellitus, type of donor and terminal creatinine. Recipient information included: sex, age, ethnicity, blood type, type of graft received, panel reactive antibody (PRA), and number of transplants (Table 1). EGL was defined as the occurrence of renal artery or vein thrombosis, ACR, AMR, or PNF within the first 90 days post-transplantation.

Table 1. Recipient and Donor Demographics - Single Center.

\begin{tabular}{|c|c|c|c|}
\hline Recipient Factors & EGL & Control & Sig. \\
\hline Age at Transplant (yrs) & 56.8 & 52 & 0.073 \\
\hline Elderly (>65 yrs) & $7(23.3 \%)$ & $115(17.8 \%)$ & 0.465 \\
\hline Male sex (n,\%) & $21(70 \%)$ & $408(62.3 \%)$ & 0.562 \\
\hline White (n,\%) & $18(60 \%)$ & $461(71.4 \%)$ & 0.217 \\
\hline $\mathrm{AA}(\mathrm{n}, \%)$ & $8(26.7 \%)$ & $144(22.3 \%)$ & 0.654 \\
\hline Hispanic (n,\%) & $3(10 \%)$ & $28(4.3 \%)$ & 0.153 \\
\hline Asian (n,\%) & $1(3.3 \%)$ & $13(2 \%)$ & 0.474 \\
\hline Retransplant (n,\%) & $9(30 \%)$ & $172(26.6 \%)$ & 0.676 \\
\hline PRA $>20 \%(n, \%)$ & $7(23.3 \%)$ & $118(18.5 \%)$ & 0.477 \\
\hline Donor Factors & EGL & Control & Sig. \\
\hline CAD (n,\%) & $25(83.3 \%)$ & $476(73.7 \%)$ & 0.291 \\
\hline $\operatorname{ECD}(\mathrm{n}, \%)$ & $11(44 \%)$ & $49(10.3 \%)$ & $\mathrm{p}<0.001$ \\
\hline $\operatorname{DCD}(n, \%)$ & $1(4.2 \%)$ & $48(10.1 \%)$ & 0.496 \\
\hline Donor age (yrs) & 46.5 & 38.3 & 0.003 \\
\hline Donor HTN (n,\%) & $13(46.4 \%)$ & $123(19.1 \%)$ & 0.001 \\
\hline Donor DM (n,\%) & $4(14.3 \%)$ & $35(5.4 \%)$ & 0.075 \\
\hline Donor cancer (n,\%) & $1(3.6 \%)$ & $16(2.5 \%)$ & 0.521 \\
\hline Smoker Donor (n,\%) & $8(27.6 \%)$ & $156(24.3 \%)$ & 0.662 \\
\hline Alcoholic Donor (n,\%) & $6(25 \%)$ & $83(17.5 \%)$ & 0.41 \\
\hline Donor BMI (kg/m2) & 30 & 26.9 & 0.004 \\
\hline KDPI & 60.4 & 38.9 & 0.001 \\
\hline Terminal Creatinine (mg/dL) & 1.2 & 0.955 & 0.034 \\
\hline CIT (hrs) & 13.9 & 11.5 & 0.629 \\
\hline WIT (hrs) & 0.83 & 1.02 & 0.196 \\
\hline
\end{tabular}

Prior to transplantation, patient profiles were cross-matched for $\mathrm{T}$ and B cell status via flow cytometry. All patients included in our study were negative cross-matches for both $\mathrm{T}$ and $\mathrm{B}$ cells. All cases of acute rejection were biopsy-proven.

Patients were pretreated with $25 \mathrm{mg}$ of diphenhydramine intravenously (IV). At the time of the procedure, induction immunosuppression with methylprednisolone $500 \mathrm{mg}$ (IV) (SoluMedrol, Pfizer, New York, NY), mycophenolate sodium $540 \mathrm{mg}$ by mouth (PO) (Myfortic, Novartis Pharmaceuticals, Basel, Switzerland) and ALE $30 \mathrm{mg}$ IV was administered.

The post-operative steroid taper consisted of: methylprednisolone $250 \mathrm{mg}$ IV on post-operative day 1, methylprednisolone $125 \mathrm{mg}$ IV on post-operative day 2, prednisone $60 \mathrm{mg}$ PO on post-operative day 3 , prednisone $40 \mathrm{mg} \mathrm{PO}$ on post-operative day 4 , and, finally, prednisone $20 \mathrm{mg}$ PO on post-operative day 5. Patients at high-risk for rejection were continued indefinitely on prednisone 5-10 mg PO.

Starting on post-operative day 1, Tacrolimus $1.5 \mathrm{mg}$ PO (Prograf, Astellas Pharma, Tokyo, Japan) and mycophenolate sodium $540 \mathrm{mg}$ PO twice per day were given. Tacrolimus levels were measured and titrated to the correct dose. Side effects permitting, mycophenolate sodium was increased to $720 \mathrm{mg}$ PO at discharge.

Antimicrobial prophylaxis was started post-operatively with sulfamethoxazole (800 mg)-trimethoprim (160 mg) 1 tab PO (Bactrim DS, AR Scientific, Philadelphia, PA) 3 times per week and clotrimazole troche $10 \mathrm{mg}$ dissolved in the mouth 4 times per day following oral care. In the event of a cytomegalovirus (CMV) mismatch, daily valgancyclovir $450 \mathrm{mg}$ PO (Valcyte, Hoffman-La Roche, Basel, Switzerland) was prescribed. The diagnosis of CMV mismatch was made by determining the IgM antibodies to CMV by polymerase chain reaction $(\mathrm{PCR})$.

Continuous variables were presented in medians and were compared using t-tests or the Mann-Whitney $U$ test when appropriate. Categorical variables including gender, ethnicity, education level, delayed graft function, and early rejection were presented in terms of percentage of the total number within the group. They were compared with Pearson's Chi-square or Fisher's Exact Test. Multivariate survival analysis was done using Cox regression analysis with multivariate factors selected from univariate results and with patient ethnicity included for comparison. Type I error level was set at 0.05 . All statistical analyses for single center were conducted using IBM SPSS ver23 (IBM Corp., Armonk, NY) and analyses for UNOS data were conducted using Stata ver14 (StataCorp LLC, College Station, TX).

\section{Results \\ UNOS database}

The rate of EGL in the UNOS database was 1,903 (3.35\%) out of 56,883 patients. Increased age, elevated BMI, and recipient diabetes were significantly associated with higher rates of EGL due to patient death when compared to the non-EGL group ( $<<0.001, \mathrm{p}=0.0220$, and $\mathrm{p}<0.001$, respectively) (Table 2 ). Recipients on the waitlist for longer periods of time had a significantly higher incidence of EGL due to patient death compared to the non-EGL group $(\mathrm{p}=0.0019)$ (Table 2). Patients who were in the ICU or hospital at the time of transplant experienced EGL due to patient death at a significantly higher rate compared to the non-EGL group $(\mathrm{p}=0.012)$ (Table 2 ). Upon multivariate analysis, only recipient age was a significant predictor of EGL due to patient death (HR: 1.04, CI: 1.03-1.04, $\mathrm{p}<0.001$ ). 
Table 2. Comparison of Recipient Characteristics between EGL-Patient Death and NonEGL.

\begin{tabular}{|l|l|l|l|}
\hline Recipient Characteristics & EGL-Pt death & Non-EGL & P-value \\
\hline Age at Tx, mean (SD) & $59.21(11.48)$ & $52.11(13.55)$ & 0.000 \\
\hline Male, n (\%) & $590(63.85 \%)$ & $33,488(60.91 \%)$ & 0.069 \\
\hline BMI, mean (SD) & $28.54(5.35)$ & $28.13(5.37)$ & 0.0220 \\
\hline Diabetes, n (\%) & $487(52.71 \%)$ & $21,847(39.75 \%)$ & 0.000 \\
\hline Causes of kidney disease & & & \\
\hline HCV, n (\%) & $60(6.49 \%)$ & $2,867(5.21 \%)$ & 0.083 \\
\hline HBV, n (\%) & $86(9.31 \%)$ & $4,553(8.28 \%)$ & 0.262 \\
\hline Days on Waiting List & $1016.45(785.25)$ & $936.71(774.01)$ & 0.0019 \\
\hline Status / Location at time of Tx, n(\%) & & & \\
\hline ICU & $3(0.32 \%)$ & $45(0.08 \%)$ & 0.012 \\
\hline Hospitalized & $11(1.19 \%)$ & $392(0.71 \%)$ & 0.089 \\
\hline Home & $866(93.72 \%)$ & $50,578(91.99 \%)$ & 0.054 \\
\hline HLA mismatch level, mean (SD) & $4.12(1.54)$ & $4.08(1.58)$ & 0.4239 \\
\hline Graft failure causes, $\mathrm{n}(\%)$ & & & \\
\hline Primary nonfunction & $33(3.57 \%)$ & $47(0.09 \%)$ & 0.000 \\
\hline Acute rejection & $20(2.16 \%)$ & $622(1.13 \%)$ & 0.003 \\
\hline Vascular Thrombosis & $30(3.25 \%)$ & $32(0.06 \%)$ & 0.000 \\
\hline
\end{tabular}

Incidence of EGL due to thrombosis was significantly more common in males and in recipients without diabetes $(\mathrm{p}=0.004$ and $\mathrm{p}=0.017$, respectively) (Table 3 ). Patients who were at home directly prior to transplantation experienced EGL due to thrombosis at a significantly higher rate compared to the non-EGL group $(\mathrm{p}=0.045)$ (Table 3). Multivariate analysis revealed no significant predictors of EGL due to thrombosis.

EGL due to PNF was significantly more common in female recipients and recipients without diabetes $(\mathrm{p}=0.004$ and $\mathrm{p}=0.01$, respectively) (Table 4). Recipient age was found to be marginally protective for recipients with EGL due to PNF (HR: 0.99, CI: 0.98-0.99, $\mathrm{p}=0.039)$. EGL due to AR was significantly more common in recipients with elevated BMI $(p=0.0003)$ (Table 5). EGL due to AR was also significantly more common in recipients who had longer times spent on the waitlist ( $1193.52 \pm 964.64$ days vs. $936 \pm 774.01$ days; $\mathrm{p}=0.0004)$ (Table 5). Previous kidney transplant (HR: 2.12, CI: 1.32-3.42, $\mathrm{p}=0.002$ ) and recipient age (HR: 1.03, CI:1.02-1.05, $\mathrm{p}<0.001$ ) were risk factors for EGL due to AR according to multivariate analysis.

Donor characteristics can be found in Table 6. Donor age was significantly increased in recipients who experienced EGL due to death $(44.04 \pm 15.12$ years; $\mathrm{p}<0.0001)$, EGL due to PNF (41.76 \pm 13.66 years; $\mathrm{p}=0.0021)$, and $\mathrm{EGL}$ due to $\mathrm{AR}(43.62 \pm 14.64$ years; $\mathrm{p}=0.0016)$ compared to the non-EGL group $(39.43 \pm 14.28$ years). Donor BMI was also significantly elevated in recipients who experienced EGL due to death $\left(28.93 \pm 7.08 \mathrm{~kg} / \mathrm{m}^{2} ; \mathrm{p}<0.0001\right)$, EGL due to thrombosis $\left(28.93 \pm 7.08 \mathrm{~kg} / \mathrm{m}^{2} ; \mathrm{p}=0.0324\right)$, and EGL due to PNF $(28.68 \pm 7.30$ $\left.\mathrm{kg} / \mathrm{m}^{2} ; \mathrm{p}=0.0216\right)$ compared to the non-EGL group $(27.88 \pm 6.53 \mathrm{~kg} /$ $\mathrm{m}^{2}$ ). DCD donors were used significantly more often in recipients who experienced EGL due to death $(18.07 \%$; $\mathrm{p}=0.032)$, EGL due to thrombosis (19.88\%; $\mathrm{p}=0.03)$, and EGL due to PNF $(20.45 \%$; $\mathrm{p}=0.01)$ compared to the non-EGL group (15.49\%). ECD donors were used significantly more often in recipients who experienced EGL due to PNF (18.49\%; $\mathrm{p}=0.05)$ and EGL due to AR (25\%; $\mathrm{p}=0.002)$ compared to the non-EGL group (14.81\%). Warm ischemia time (WIT) was significantly longer in recipients who experienced EGL due to death $(23.50 \pm 16.54 \mathrm{~min} ; \mathrm{p}<0.0001)$, and EGL due to PNF $(24.41 \pm 22.72$ min; $\mathrm{p}=0.0335)$ compared to the non-EGL group $(20.82 \pm 13.97 \mathrm{~min})$. Cold ischemia time (CIT) was significantly longer in recipients who experienced EGL due to death $(23.5 \pm 16.54$ hrs.; $\mathrm{p}=0.0001)$, EGL due
Table 3. Comparison of Recipient Characteristics between EGL-Thrombosis and NonEGL.

\begin{tabular}{|l|l|l|l|}
\hline Recipient Characteristics & EGL-Thrombosis & Non-EGL & P-value \\
\hline Age at Tx, mean (SD) & $50.96(14.23)$ & $52.11(13.55)$ & 0.1310 \\
\hline Male, $\mathrm{n}(\%)$ & $171(53.11 \%)$ & $33,488(60.91 \%)$ & 0.004 \\
\hline BMI, mean (SD) & $28.29(5.52)$ & $28.13(5.37)$ & 0.5947 \\
\hline Diabetes, $\mathrm{n}(\%)$ & $107(32.23 \%)$ & $21,847(39.74 \%)$ & 0.017 \\
\hline Causes of kidney disease & & & \\
\hline HCV, n (\%) & $13(4.04 \%)$ & $2,867(5.21 \%)$ & 1.000 \\
\hline HBV, n (\%) & $21(6.52 \%)$ & $4,553(8.28 \%)$ & 0.253 \\
\hline Days on Waiting List & $877.16(710.15)$ & $936.71(774.01)$ & 0.1685 \\
\hline Status / Location at time of Tx, n (\%) & & & \\
\hline ICU & $0(0 \%)$ & $45(0.08 \%)$ & 0.608 \\
\hline Hospitalized & $4(1.24 \%)$ & $392(0.71 \%)$ & 0.261 \\
\hline Home & $306(95.03 \%)$ & $50,578(91.99 \%)$ & 0.045 \\
\hline HLA mismatch level, mean (SD) & $4.04(1.57)$ & $4.08(1.58)$ & 0.3209 \\
\hline Graft failure causes, $\mathrm{n}(\%)$ & & & \\
\hline Primary nonfunction & $322(100 \%)$ & $47(0.09 \%)$ & 0.000 \\
\hline Acute rejection & $0(0 \%)$ & $622(1.13 \%)$ & 0.055 \\
\hline Vascular Thrombosis & - & $32(0.06 \%)$ & - \\
\hline
\end{tabular}

Table 4. Comparison of Recipient Characteristics between EGL-PNF and Non-EGL.

\begin{tabular}{|l|l|l|l|}
\hline Recipient Characteristics & EGL-PNF & Non-EGL & P-value \\
\hline Age at Tx, mean (SD) & $51.43(14.12)$ & $52.11(13.55)$ & 0.3479 \\
\hline Male, $\mathrm{n}(\%)$ & $191(53.50 \%)$ & $33,488(60.91 \%)$ & 0.004 \\
\hline BMI, mean (SD) & $28.53(5.67)$ & $28.13(5.37)$ & 0.1588 \\
\hline Diabetes, $\mathrm{n}(\%)$ & $118(33.05 \%)$ & $21,847(39.74 \%)$ & 0.010 \\
\hline Causes of kidney disease & & & \\
\hline HCV, $\mathrm{n}(\%)$ & $14(3.92 \%)$ & $2,867(5.21 \%)$ & 0.273 \\
\hline HBV, $\mathrm{n}(\%)$ & $21(5.88 \%)$ & $4,553(8.28 \%)$ & 0.101 \\
\hline Days on Waiting List, mean (SD) & $891.79(726.94)$ & $936.71(774.01)$ & 0.2743 \\
\hline Status / Location at time of Tx, $\mathrm{n}(\%)$ & & & \\
\hline ICU & $0(0 \%)$ & $45(0.08 \%)$ & 0.589 \\
\hline Hospitalized & $4(1.12 \%)$ & $392(0.71 \%)$ & 0.363 \\
\hline Home & $334(93.56 \%)$ & $50,578(91.99 \%)$ & 0.278 \\
\hline HLA mismatch level, mean (SD) & $4.08(1.57)$ & $4.08(1.58)$ & 0.9446 \\
\hline Graft failure causes, $\mathrm{n}(\%)$ & & & \\
\hline Primary nonfunction & - & $47(0.09 \%)$ & - \\
\hline Acute rejection & $0(0 \%)$ & $622(1.13 \%)$ & 0.043 \\
\hline Vascular Thrombosis & $322(90.20 \%)$ & $32(0.06 \%)$ & 0.000 \\
\hline
\end{tabular}

Table 5. Comparison of recipient characteristics between EGL-acute rejection and nonEGL.

\begin{tabular}{|l|l|l|l|}
\hline Recipient Characteristics & $\begin{array}{l}\text { EGL-Acute } \\
\text { Rejection }\end{array}$ & Non-EGL & P-value \\
\hline Age at Tx, mean (SD) & $53.20(13.66)$ & $52.11(13.55)$ & 0.3865 \\
\hline Male, $\mathrm{n}(\%)$ & $80(68.97 \%)$ & $33,488(60.91 \%)$ & 0.076 \\
\hline BMI, mean (SD) & $29.95(5.74)$ & $28.13(5.37)$ & 0.0003 \\
\hline Diabetes, $\mathrm{n}(\%)$ & $40(34.48 \%)$ & $21,847(39.74 \%)$ & 0.248 \\
\hline Causes of kidney disease & & & \\
\hline HCV, n (\%) & $13(11.21 \%)$ & $2,867(5.21 \%)$ & 0.004 \\
\hline HBV, n (\%) & $12(10.34 \%)$ & $4,553(8.28 \%)$ & 0.421 \\
\hline Days on Waiting List, mean (SD) & $1193.52(964.64)$ & $936.71(774.01)$ & 0.0004 \\
\hline Status / Location at time of Tx, n(\%) & & & \\
\hline ICU & $0(0 \%)$ & $45(0.08 \%)$ & 0.758 \\
\hline Hospitalized & $1(0.86 \%)$ & $392(0.71 \%)$ & 0.849 \\
\hline Home & $112(96.55 \%)$ & $50,578(91.99 \%)$ & 0.071 \\
\hline HLA mismatch level, mean (SD) & $4.08(1.66)$ & $4.08(1.58)$ & 0.9789 \\
\hline Graft failure causes, $\mathrm{n}(\%)$ & & & \\
\hline Primary nonfunction & $0(0 \%)$ & $47(0.09 \%)$ & 0.753 \\
\hline Acute rejection & - & $622(1.13 \%)$ & - \\
\hline Vascular Thrombosis & $0(0 \%)$ & $32(0.06 \%)$ & 0.795 \\
\hline
\end{tabular}


Table 6. Comparison of donor characteristics between EGL and non-EGL.

\begin{tabular}{|c|c|c|c|c|c|}
\hline Donor Characteristics & EGL-Pt. Death & EGL-Thrombosis & EGL-PNF & EGL-Acute Rejection & Non-EGL \\
\hline Age, mean (SD), p-value & $44.04(15.12), 0.0000$ & 40.61 (13.63), 0.1390 & 41.76 (13.66), 0.0021 & 43.62 (14.64), 0.0016 & $39.43(14.28)$ \\
\hline Male, $\mathrm{n}(\%), p$-value & $572(61.90 \%), 0.667$ & $187(58.07 \%), 0.250$ & $210(58.82 \%), 0.356$ & $73(62.93 \%), 0.704$ & $33,653(61.21 \%)$ \\
\hline \multicolumn{6}{|l|}{ Race, $\mathrm{n}(\%), p$-value } \\
\hline $\mathrm{AA}$ & $141(5.26 \%), 0.191$ & $41(12.73 \%), 0.592$ & $47(13.17 \%), 0.743$ & $16(13.79 \%), 0.993$ & $7,568(13.77 \%)$ \\
\hline White & $610(66.02 \%), 0.101$ & $232(72.05 \%), 0.177$ & $256(71.71 \%), 0.199$ & $78(67.24 \%), 0.763$ & $37,685(68.54 \%)$ \\
\hline Hispanic & $134(14.50 \%), 0.333$ & $39(12.11 \%), 0.496$ & $43(12.04 \%), 0.451$ & $15(12.93 \%), 0.881$ & $7,371(13.41 \%)$ \\
\hline Asian & $23(2.49 \%), 0.925$ & $7(2.17 \%), 0.757$ & $7(1.96 \%), 0.558$ & $3(2.59 \%), 0.919$ & $1,342(2.44 \%)$ \\
\hline Other & $16(1.73 \%), 0.801$ & $3(0.93 \%), 0.224$ & $4(1.12 \%), 0.310$ & $4(3.45 \%), 0.200$ & $1,014(1.84 \%)$ \\
\hline BMI, mean (SD), p-value & $28.93(7.08), 0.0000$ & $28.67(7.34), 0.0324$ & $28.68(7.30), 0.0216$ & $27.79(6.50), 0.8784$ & $27.88(6.53)$ \\
\hline Creatinine, mean (SD), $p$-value & $1.14(0.67), 0.2716$ & $1.14(0.68), 0.5358$ & $1.17(0.71), 0.9684$ & $1.09(0.54), 0.3426$ & $1.17(0.94)$ \\
\hline Bilirubin, mean (SD), p-value & $0.96(1.32), 0.3717$ & 0.83 (1.09), 0.0325 & $0.88(1.31), 0.0887$ & $1.02(0.64), 0.9219$ & $1.01(1.44)$ \\
\hline DRI, mean (SD), p-value & 42.20 (116.72), 0.9755 & $49.30(118.51), 0.2252$ & 49.71 (117.62), 0.1771 & 52.14 (133.18), 0.3091 & $42.09(106.28)$ \\
\hline DCD, $\mathrm{n}(\%), p$-value & $167(18.07 \%), 0.032$ & $64(19.88 \%), 0.030$ & $73(20.45 \%), 0.010$ & $19(16.38 \%) 0.793$ & $8,519(15.49 \%)$ \\
\hline $\mathrm{ECD}, \mathrm{n}(\%), p$-value & $252(27.27 \%), 0.000$ & $54(16.77 \%), 0.325$ & $66(18.49 \%), 0.052$ & $29(25.00 \%), 0.002$ & $8,145(14.81 \%)$ \\
\hline $\begin{array}{l}\text { Warm ischemia time in } \\
\text { minutes, mean (SD), } p \text {-value }\end{array}$ & $23.50(16.54), 0.0168$ & $24.16(13.97), 0.0628$ & $24.41(22.72), 0.0335$ & $17.58(9.41), 0.3115$ & $20.82(13.97)$ \\
\hline $\begin{array}{l}\text { Cold ischemia time in hours, } \\
\text { mean (SD), } p \text {-value }\end{array}$ & $18.01(9.14), 0.0001$ & $18.82(8.66), 0.0001$ & $18.73(8.75), 0.0001$ & $16.50(8.72), 0.6591$ & $16.87(8.93)$ \\
\hline
\end{tabular}

to thrombosis $(18.82 \pm 8.66 \mathrm{hrs.;} \mathrm{p}=0.0001)$, and EGL due to PNF $(18.73$ \pm 8.75 hrs.; $\mathrm{p}=0.0001)$ compared to recipients who did not experience EGL $(16.87 \pm 8.93 \mathrm{hrs})$.

\section{Single center}

A total of 676 patients underwent kidney transplantation at our institution during the study period. 30 (4.4\%) patients had EGL (Table 7). Recipients of expanded criteria donor (ECD) grafts had a significantly higher rate of EGL compared to controls (44\% and $10.3 \%$, respectively; $\mathrm{p}<0.001$, Table 1 ). Patients who experienced EGL received grafts from significantly older donors compared to controls ( 46.5 years and 38.3 years, respectively; $\mathrm{p}=0.003$, Table 1 ). Donor body mass index (BMI) was significantly higher in recipients with EGL compared to controls $\left(30 \mathrm{~kg} / \mathrm{m}^{2}\right.$ and $26.9 \mathrm{~kg} / \mathrm{m}^{2}$, respectively; $\mathrm{p}=0.004$, Table 1$)$. Recipients with EGL received grafts from donors with significantly higher Kidney Donor Profile Indices (KDPI) compared to controls (60.4 and 38.9, respectively; $\mathrm{p}=0.001$, Table 1 ). All other recipient and donor demographics are listed in Table 1.

Graft loss due to graft thrombosis was significantly higher in recipients with EGL compared to controls $(24.1 \%$ and $0.7 \%$, respectively; $\mathrm{p}<0.05)$. Graft loss because of AR was not significantly different in the EGL group compared to controls (13.8\% and $15.2 \%$, respectively; $\mathrm{p}>0.05)$. The rate of death as a cause of graft loss did not differ significantly between recipients with EGL and controls $(36.7 \%$ and $46.9 \%$, respectively; $\mathrm{p}>0.05$; Table 7 ).

1-, 3-, and 5-year patient survival was significantly lower in recipients with EGL compared to controls $(63 \%, 50 \%, 50 \%$ and $97.5 \%$, $91.4 \%, 86 \%$, respectively; all $\mathrm{p}<0.001$ ) (Table 8 ). There was no significant difference in the rate of rejection between recipients with EGL and controls ( $33.3 \%$ and $26.6 \%$, respectively; $\mathrm{p}=0.406$ ). The median number of days until rejection in the EGL group was significantly less than controls ( 18.8 days and 308.4 days, respectively; $\mathrm{p}=0.001$ ). The median number of days until the death for those with EGL was significantly less than controls (327.9 days and 1145.8 days, respectively; $\mathrm{p}<0.001$ ). Multivariate analysis suggested that $\mathrm{ECD}(\mathrm{HR}=5.163, \mathrm{CI}=2.217-12.027$, $\mathrm{p}<0.001)$ and donor BMI $(\mathrm{HR}=1.148, \mathrm{CI}=1.069-1.232, \mathrm{p}<0.001)$ were significant predictors of EGL.

\section{Discussion}

EGL after kidney transplantation is a relatively rare, yet catastrophic event, occurring at rates of $4.4 \%$ at our center and $3.35 \%$ among UNOS
Table 7. Cause of graft failure - single center

\begin{tabular}{|l|l|l|l|l|}
\hline Cause & $4(13.8 \%)$ & $22(15.2 \%)$ & DGF & \begin{tabular}{l} 
Control \\
\hline Acute rejection \\
Rejection
\end{tabular} \\
\hline Chronic rejection & 0 & $21(14.5 \%)^{*}$ & $2(13.3 \%)$ & $6(20.7 \%)$ \\
\hline Graft thrombosis & $7(24.1 \%)$ & $1(0.7 \%)^{*}$ & $1(6.7 \%)$ & $4(13.8 \%)$ \\
\hline Other allograft complication & $1(3.4 \%)$ & $1(0.7 \%)$ & $0^{*}$ & $1(3.4 \%)^{*}$ \\
\hline Death & $11(36.7 \%)$ & $68(46.9 \%)$ & $0^{*}$ & 0 \\
\hline Recurrent disease & 0 & $9(6.2 \%)$ & $8(53.3 \%)$ & $7(24.1 \%)$ \\
\hline PNF & $7(24.1 \%)$ & $0^{*}$ & $1(6.7 \%)$ & $2(6.9 \%)$ \\
\hline Other & 0 & $23(15.9 \%)^{*}$ & $0^{*}$ & $0^{*}$ \\
\hline $\begin{array}{l}*=\text { Significant difference } \\
\text { at p }<0.05, \text { either to control } \\
\text { (EGL) or to EGL (DGF, } 90\end{array}$ & & & & \\
day rejection) & & & $3(20 \%)^{*}$ & $9(31 \%)^{*}$ \\
\hline
\end{tabular}

Table 8. Negative outcomes for EGL and comparison groups - single center.

\begin{tabular}{|l|l|l|l|}
\hline Factor & EGL & Control & Sig \\
\hline Patient survival & $15(50 \%)$ & $557(86.2 \%)$ & $\mathbf{p}<\mathbf{0 . 0 0 1}$ \\
\hline- 1 year & $17(63 \%)$ & $594(97.5 \%)$ & $\mathbf{p}<\mathbf{0 . 0 0 1}$ \\
\hline - 3 year & $10(50 \%)$ & $417(91.4 \%)$ & $\mathbf{p}<\mathbf{0 . 0 0 1}$ \\
\hline-5 year & $10(50 \%)$ & $313(86 \%)$ & $\mathbf{p}<\mathbf{0 . 0 0 1}$ \\
\hline Rejection rate & $10(33.3 \%)$ & $172(26.6 \%)$ & $\mathbf{0 . 4 0 6}$ \\
\hline Median Survival & EGL & Control & Sig \\
\hline Rejection-free & 14.9 & 1340.7 & $\mathbf{p}<\mathbf{0 . 0 0 1}$ \\
\hline Patient & 1067.6 & 1743.9 & $\mathbf{p}<\mathbf{0 . 0 0 1}$ \\
\hline Days until & EGL & Control & Sig \\
\hline Rejection & 18.8 & 308.4 & $\mathbf{0 . 0 0 1}$ \\
\hline Death & 327.9 & 1145.8 & $\mathbf{p}<\mathbf{0 . 0 0 1}$ \\
\hline
\end{tabular}

patient data. Thus, it is important to understand the risk factors associated with EGL in order to better inform clinical decision-making. We defined EGL as renal artery or vein thrombosis, ACR, AMR, PNF, or recipient death occurring within 90 days of kidney transplantation. Other reports defined EGL as occurring within 30 days of kidney transplantation $[4,5]$. We chose a 90 -day window because any patients who lost their allograft during this time period would be able to retain their pre-transplantation listing time.

The limited, single-center analyses that exist on EGL to date have suggested that increased donor age, DCD donor type, ECD donor type, and increased CIT were associated with EGL [4,5]. Cox regression analysis of our single-center data confirmed that ECD donor type was 
a significant predictor of EGL. The results of the review of UNOS data, however, were not as straightforward. Our data suggest that increased donor age is significantly associated with only EGL due to patient death, PNF, and AR. DCD donor type is associated with EGL due to patient death, thrombosis, and PNF, but not AR. Finally, ECD donor type is significantly associated with EGL due to patient death and AR. Multivariate analysis revealed, however, that none of these factors was a significant predictor of EGL vs. non-EGL. In this regard, the results of our UNOS review differ from those of both our own single-center review and those of Hamed et al. and Phelan et al. [4,5].

Given that data on EGL is limited, we sought to compare the potential risk factors presented in our data to those reported for thrombosis, AR, PNF, and patient death outside the scope of EGL to determine how our findings, in the context of EGL, compare.

Many have reported increased donor age as a risk factor for renal allograft thrombosis [6-8]. In the context of EGL, our data indicate that there was no significant difference in donor age between recipients who did not experienced EGL and those who experienced EGL due to thrombosis. A major risk factor for graft thrombosis is a history of thrombosis [4,7]. Unfortunately, our data did not include whether or not recipients had a history of thrombosis thus preventing us from drawing additional conclusions.

Lebranchu et al. reported that older donor age was a significant predictor of AR following transplantation. Our results correlate with their findings and further, suggest that in the context of EGL, increased donor age is also associated with an increased risk for recipient death and PNF. They also suggested that African American ethnicity and extended CIT were related to increased rates of AR. Our data, however, suggest that there was no significant difference in the number of African American recipients who experienced AR as a cause of EGL and those who did not. Additionally, there was no significant difference in CIT between the two groups. Delayed graft function is a well-known risk factor for AR $[9,10]$. Unfortunately, we did not have data on the recipients who experienced delayed graft function.

Hamed et al. [4] found an increased incidence of PNF in DCD kidneys, which they believed was due to warm ischemic injury as these kidneys are more vulnerable to ischemic reperfusion injury. Similarly, Snoejis, et al. noted that patients who suffered from PNF typically had impaired hemodynamic status during the time of transplant [11]. During the surgery, these patients have a lower BP than those with delayed or immediate graft function. Our data supports these findings as an increase in WIT and CIT can increase the risk of EGL. Our results along with these studies demonstrate that PNF may reflect the quality of the donor organ more than the recipient.

Regarding patient mortality, Debout et al. demonstrated a significant proportional increase in recipient mortality for each additional hour of CIT. We report significantly longer CIT in recipients who experienced EGL due to patient death. CIT was also significantly greater in recipients who experienced EGL due to thrombosis and acute rejection. It has also been reported that recipients of ECD grafts have a higher risk of death in the early post-transplant period [12]. Our data are similar, recipients who suffered EGL due to death were significantly more likely to have received an ECD graft.

In recipients who experience EGL, it is important to know what happened to them after graft loss. In analyzing the post-EGL outcomes of patients at our own institution, we found 20 recipients who experienced EGL attributable to causes other than patient death. Of these, 3 were re-transplanted and 3 died while waiting for a re-transplant. The remaining 14 are still awaiting re-transplant to our knowledge.
Our analysis has a number of strengths. Primarily, it includes data from both a single center (similar to previous analyses on EGL) and the UNOS database. Furthermore, we were able to identify risk factors specific to certain causes of EGL using the UNOS data. Finally, we utilized a relatively large sample size. One weakness was the lack of certain data points, such as previous history of thrombosis. Additionally, the relative lack of other reports on the topic of EGL make it difficult to compare out conclusions to others [13-17].

\section{Conclusion}

The rates of EGL were $4.4 \%$ at our center and 3.35\% among UNOS patient data. Although an obvious trend is difficult to discern, our data generally suggest that sub-optimal allografts are associated with an increase in EGL due to a variety of factors. Given these results, we believe transplant providers should use caution and increased monitoring of recipients receiving what could be considered suboptimal allografts.

\section{References}

1. Michael Gibson C, Korjian S, Tricoci P, Daaboul Y, Yee M, et al. (2016) Safety and Tolerability of CSL112, a Reconstituted, Infusible, Plasma-Derived Apolipoprotein A-I, After Acute Myocardial Infarction: The AEGIS-I Trial (ApoA-I Event Reducing in Ischemic Syndromes I). Circulation 134: 1918-1930. [Crossref]

2. OPTN/SRTR Annual Report 2012. https://optn.transplant.hrsa.gov/data/view-datareports/annual-report/

3. Perico N, Ruggenenti P, Scalamogna M, Remuzzi G (2003) Tackling the shortage of donor kidneys: how to use the best that we have. Am J Nephrol 23: 245-259.

4. Hamed MO, Chen Y, Pasea L, Watson CJ, Torpey N, et al. (2015) Early graft loss after kidney transplantation: risk factors and consequences. Am J Transplant 15: 1632-1643. [Crossref]

5. Phelan P, O'Kelly P, Tarazi M (2012) Renal allograft loss in the first post-operative month: causes and consequences. Clin Transplant 26: 544-549.

6. Ponticelli C, Moia M, Montagnino G (2009) Renal allograft thrombosis. Nephrology Dialysis Transport 24(5): 1388-1393.

7. Keller AK, Jorgensen TM, Jespersen B (2012) Identification of risk factors for vascular thrombosis may reduce early renal graft loss: a review of recent literature. $J$ Transplant 2012: 793461. [Crossref]

8. Luna E, Cerezo I, Collado G (2010) Vascular thrombosis after kidney transplantation predisposing factors and risk index. Transplant Proc 42 (8): 2928-2930.

9. Lebranchu Y, Baan C, Biancone L (2013) Pretransplant identification of acute rejection risk following kidney transplantation. Transplant International 27 (2): 129-138.

10. Wu WK, Famure O, Li Y, Kim SJ (2015) Delayed graft function and the risk of acute rejection in the modern era of kidney transplantation. Kidney International 88 (4): 851-858.

11. Snoeijs MGJ, Wiermans B, Christiaans MH (2007) Recipient hemodynamics during non-heart-beating donor kidney transplantation are major predictors of primary nonfunction. AMJ Transplant 7 (5): 1158-1166.

12. Debout A, Foucher Y, Trebern-Launay K (2015) Each additional hour of cold ischemia time significantly increases the risk of graft failure and mortality following renal transplantation. Kidney International 87 (2): 343-349.

13. Gill JS, Schaeffner E, Chadban S, Dong J, Rose C, et al. (2013) Quantification of the early risk of death in elderly kidney transplant recipients. Am J Transplant 13: 427-432. [Crossref]

14. Meier-Kriesche H, Arndorfer J, Kaplan B (2002) The impact of body mass index on renal transplant outcomes: a significant independent risk factor for graft failure and patient death. Transplantation 73 (1): 70-74.

15. Meier-Kriesche H, and Kaplan B (2002) Waiting time on dialysis as the strongest modifiable risk factor for renal transplant outcomes: a paired donor kidney analysis. Transplantation 74 (10): 1377-1381.

16. McAdams-DeMarco MA, Law A, King E, Orandi B, Salter M, et al. (2015) Frailty and mortality in kidney transplant recipients. Am J Transplant 15: 149-154. [Crossref]

Copyright: (C2017 Brooks JT. This is an open-access article distributed under the terms of the Creative Commons Attribution License, which permits unrestricted use, distribution, and reproduction in any medium, provided the original author and source are credited. 